\title{
Practical Experiences of Delivering Multidrug-Resistant Tuberculosis Comprehensive Supportive Care Services in China
}

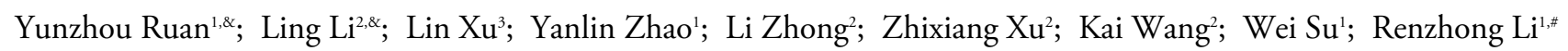

\begin{abstract}
Summary
What is already known about this topic?

The World Health Organization consolidated guidelines on recommend care for tuberculosis (TB) and support for multidrug-resistant TB (MDR-TB) patients. But guidelines have not provided detailed guidance or tools for health services providers to implement comprehensive patient care.

What is added by this report?

China CDC and the United States Agency for International Development-funded Control and Prevention of MDR-TB program introduced a differentiated and personalized comprehensive and supportive care services (CSC) to improve treatment adherence.

What are the implications for public health practice?

The CSC model helps MDR-TB patients complete treatment and improve treatment success rates, and scaling up the program and implementation in other parts of the country is worth consideration.
\end{abstract}

\section{BACKGROUND}

According to Global Tuberculosis Report 2020 (1), the global treatment success rate for multidrugresistant tuberculosis (MDR-TB) is 57\%, far below the World Health Organization (WHO) target of curing at least $75 \%$ of MDR-TB patients. China - with a treatment success rate of $54 \%$ in 2019 - is no exception; nearly half of all treated MDR-TB patients are lost to follow-up, treatment failure, or death. Failure to support patients throughout the treatment process leads to incomplete treatment, treatment failure, and associated morbidity and mortality, and it also wastes significant financial resources the health system invested in MDR-TB diagnosis and treatment (2-3). More importantly, patients continue to suffer. They are not only at risk of developing extensive drug resistant tuberculosis (XDR-TB), but also of transmitting MDR-TB to their families and communities, increasing the incremental burden of MDR-TB patients, and fueling a growing public health crisis.

The WHO proposes that providing patient care services improves treatment adherence, helps patients complete treatment, and improves overall cure rates (4-5). However, the WHO guidelines have not provided detailed guidance or tools for national tuberculosis control programs and health services providers to implement comprehensive patient care (6-7). In 2017, China CDC and the United States Agency for International Development-funded Control and Prevention of MDR-TB program implemented by Family Health International (FHI360), introduced a differentiated and personalized comprehensive and supportive care services (CSC). The CSC framework, which was based on global MDR-TB guidelines but adapted to China's context, and included 4 thematic areas and 11 elements (8) which were piloted in selected sites in China to explore how to provide practical care services for MDR-TB patients (Table 1).

\section{PILOT SELECTION AND KEY CONTENT}

Beginning in June 2017, China CDC and FHI360 selected 6 cities (Jinan, Urumqi, Zhenjiang, Yichang, Wuhan, and Kunming) across 5 provincial-level administrative divisions (PLADs) (Shandong Province, Xinjiang Uygur Autonomous Region, Jiangsu Province, Hubei Province, and Yunnan Province) from the east to the west - chosen based on having a large number of MDR-TB patients and demonstrating good inter-agency cooperation - to pilot the CSC model. Each pilot site established a "care team" comprised of local CDC staff, clinical doctors, and nurses from designated TB hospitals, community/village physicians, and patient volunteers. They conducted a series of training on a range of topics including the following: standardized diagnosis and treatment management of adverse reactions for healthcare providers; counselling skills for nurses; community-based patient management, infection control at the households of 
TABLE 1. The supportive care services framework - thematic areas and elements.

\begin{tabular}{ll}
\hline \multicolumn{1}{c}{ Thematic areas } & \multicolumn{1}{c}{ Elements } \\
\hline $\begin{array}{l}\text { Respect for patient autonomy and support of self-efficacy } \\
\text { strengthened }\end{array}$ & $\begin{array}{l}\text { Patient intake assessment and individual care plan } \\
\text { Patient and family education on treatment } \\
\text { Support treatment and care accessible to patients }\end{array}$ \\
$\begin{array}{l}\text { Maximizing physical comfort, safety, and wellness among } \\
\text { MDR-TB patients }\end{array}$ & $\begin{array}{l}\text { Monitoring and treatment of adverse drug effects and co-morbid physical } \\
\text { conditions } \\
\text { Nutritional support }\end{array}$ \\
$\begin{array}{l}\text { Palliative care } \\
\text { Strenghtening psycho-emotional support and protection } \\
\text { from social isolation or discrimination }\end{array}$ & $\begin{array}{l}\text { Patient-centered counselling throughout care } \\
\text { Monitoring and treatment of mental health conditions } \\
\text { Support for reducing social isolation }\end{array}$ \\
Preventing catastrophic costs to patient and family & Protect patient and family from stigma and discrimination \\
strengthened & Patient cost saving or reduction activities \\
\hline
\end{tabular}

MDR-TB patients, and management of adverse reactions for community and village doctors; and treatment adherence support for volunteers.

During the pilot, providers focused on several key points over the course of treatment - this included nurses working with patients during in-hospital treatment to formulate a patient rehabilitation plan and provide support for a range of patient-centered care services including patient education and counselling for treatment decision making, taking daily medication, dealing with mild adverse reactions, providing sputum samples, and diet and nutrition. When discharged from the hospital, the local CDC system was mobilized for rapid connection of patients with community/village doctors for follow-up management. These community-based staff tracked patients to avoid loss to follow-up, and when patients went back to the hospital for check-ups, green channels were established for convenience for the patients. Lastly, medical staff and peer volunteers provided diversified social support services which included hotline calls for medical staff to answer healthcare questions, the establishment of 57 Zone patientcare social media communication groups via $Q Q$ or WeChat platforms, the Baidu $\mathrm{TB}$ online forum, provision of peer support, and volunteer services by public interest lawyers.

\section{EFFECTIVENESS AND EXPERIENCE}

The pilot has achieved remarkable results. Pilot sites provided comprehensive care services for existing and newly enrolled MDR-TB patients between July and September 2017. By the end of the pilot in March 2020, 232 MDR-TB patients had received care services for the full course, with a treatment success rate of $73 \%$ (compared to $42 \%$ for a historical cohort in 2015). Loss to follow-up decreased from $15 \%$ in 2015 to $3 \%$ during the pilot period, and self-reported satisfaction with services increased among MDR-TB patients (9). The model was also well accepted by healthcare staff and, at present, 5 pilot PLADs have become demonstration sites to replicate the CSC model across China. The national CDC established a national core team of counselling trainers with proven field experiences and ability from the pilot PLADs. They developed a series of illustrated tool kits to ensure care services were replicated with fidelity. The CSC model has been gradually rolling out and by December 2020 there were 45 prefectures and cities across 13 PLADs implementing the supportive care services for MDR-TB patients. Care services have also been incorporated into the national TB control work standards and guidelines. Finally, the practical experiences gained during the pilot project have been compiled into an operational manual, which will be published soon.

\section{KEY EXPERIENCES IN THE PILOT PROCESS AS BELOW}

The traditional lack of emphasis on patient care services should be changed. Care services are not a "nice to have" extra, they are a core component of effective, patient-centered MDR-TB treatment. However, ensuring comprehensive, patient-centered care and treatment services also requires that CDCs, designated TB hospitals, and community health institutions make effective and coordinated use of their respective advantages and strengths.

Communication and cooperation between CDCs, designated TB hospitals, and community health institutions is a precondition for launching the supportive care services. Before this model can be implemented, it is necessary to clarify respective 
responsibilities, establish a cooperative process, and optimize internal working processes among these organizations.

Training and follow-up demonstration and mentoring are key to implementing care services. As this model has been replicated, there has been a need for experienced demonstration sites and for experienced and skilled counsellors and TB clinicians. These experienced implementers can provide training and technical support and share successful innovations with the new expansion sites, whose providers can strengthen their skills in problematic areas such as case management for MDR-TB patients, patient adherence to regularly scheduled treatment monitoring visits, and compliance with the required sputum testing.

\section{CHALLENGES \& PERSPECTIVE}

Early diagnosis and standardizd treatment create a solid foundation for the success of the CSC model for MDR-TB. There remains a huge gap, however, that nearly $20 \%$ of the prefectures in China do not have the capacity to diagnose MDR-TB rapidly and approximately $25 \%$ of the patients confirmed positive for MDR-TB will not be able to receive treatment due to their inability to pay for costly anti-TB drugs. In 2019, the Chinese Government developed the 2019-2022 National Action Plan To Stop Tuberculosis with joint political commitment from 8 ministries (10), which requires that the local governments of all the prefectures should put rapid diagnosis and standardized treatment of drug resistance $\mathrm{TB}$ in place by 2022. The mandate will result in promising solutions to the existing challenges and lead to greater MDR-TB treatment success.

In the meantime, the CSC model has proven to be instrumental in bridging the following gaps at the individual patient level: 1) raising presumptive MDRTB patients' awareness for use of rapid diagnosis; 2) facilitating well-informed decision making and preparedness among the confirmed MDR-TB patients for timely diagnosis and proper treatment; and 3) providing psychosocial support to strengthen their capacity to cope with the challenges in their MDR-TB treatment to reach cure.

doi: $10.46234 / \mathrm{ccdcw} 2021.146$

\# Corresponding author: Renzhong Li, lirz@chinacdc.cn.

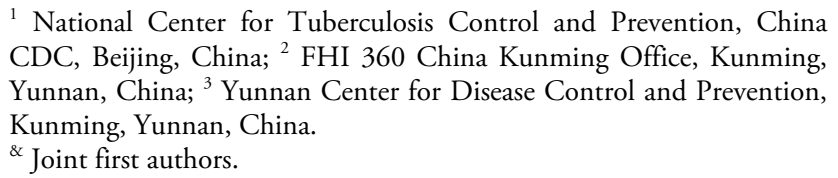

Submitted: May 18, 2021; Accepted: June 22, 2021

\section{REFERENCES}

1. World Health Organization. Global tuberculosis report 2020. 2020. https://apps.who.int/iris/bitstream/handle/10665/336069/9789240013 131-eng.pdf. [2021-1-7].

2. Alipanah N, Jarlsberg L, Miller C, Linh NN, Falzon D, Jaramillo E, et al. Adherence interventions and outcomes of tuberculosis treatment: a systematic review and meta-analysis of trials and observational studies. PLoS Med 2018;15(7):e1002595. http://dx.doi.org/10.1371/journal. pmed.1002595.

3. Munro SA, Lewin SA, Smith HJ, Engel ME, Fretheim A, Volmink J. Patient adherence to tuberculosis treatment: a systematic review of qualitative research. PLoS Med 2007;4(7):e238. http://dx.doi.org/10. 1371/journal.pmed.0040238.

4. World Health Organization. Implementing the End TB Strategy: the essentials. Geneva, Switzerland: WHO; 2015. WHO/HTM/TB/ 2015.31.

5. WHO. Consolidated guidelines on tuberculosis: module 4: treatment drug-resistant tuberculosis treatment. Geneva: World Health Organization; 2020. Licence: CC BY-NC-SA 3.0 IGO.

6. Cocozza AM, Linh NN, Jaramillo E. Is effective patient support missing in the global response to multidrug-resistant tuberculosis? Int J Tuberc Lung Dis 2020;24(6):626 - 30. http://dx.doi.org/10.5588/ijtld.19. 0529.

7. Odone A, Roberts B, Dara M, van den Boom M, Kluge H, McKee M. People- and patient-centred care for tuberculosis: models of care for tuberculosis. Int J Tuberc Lung Dis 2018;22(2):133 - 8. http://dx. doi.org/10.5588/ijtld.17.0608.

8. D'Arcy Richardson, Lisa Adams, Hilton Lam. Delivering Comprehensive Supportive Care Services to people with drug-resistant tuberculosis, United States Agency for International Development Evaluation Assignment Number: 498. 2019. https://pdf.usaid.gov/ pdf_docs/PA00TXC4.pdf.

9. Li RZ, RuanYZ, Xu L, Zhao YL. A handbook of comprehensive supportive care for people with drug-resistant tuberculosis. Beijing: People's Medical Publishing House. 2021.

10. Bureau of disease prevention and control national health commission of the People's Republic of China, national action plan to stop tuberculosis (2019-2022). http://www.nhc.gov.cn/jkj/s3589/201906/ b30ae2842c5e4c9ea2f9d5557ad4b95f.shtml. [2021-5-25]. (In Chinese). 